\title{
How to get phonon noise limited uncooled bolometers?
}

\author{
B. Guillet, S. Liu, S. Wu, L. Méchin \\ GREYC, UMR6072, CNRS-ENSICAEN-Université de \\ Caen Basse-Normandie \\ 6 boulevard Maréchal Juin \\ 14050 Caen cedex, France \\ E-mail: bruno.guillet@unicaen.fr
}

\author{
C. Adamo, D.G. Schlom* \\ Department of Materials Science and Engineering, \\ Cornell University, Ithaca, \\ NY 14853-1501, USA \\ * Kavli Institute at Cornell for Nanoscale Science, Cornell \\ University, Ithaca, New York 14853-1501, USA
}

\begin{abstract}
In this paper we present the careful study of the noise of uncooled bolometers made of free-standing epitaxial $\mathrm{La}_{0.7} \mathrm{Sr}_{0.3} \mathrm{MnO}_{3} / \mathrm{SrTiO}_{3}$ (LSMO/STO) thin films fabricated by micromachining of the silicon substrates. Relatively high values of Temperature Coefficient of Resistance, named TCR = $1 / \mathrm{R} \times \mathrm{dR} / \mathrm{dT}$ (about $0.02 \mathrm{~K}^{-1}$ ), could be achieved in LSMO at room temperature, and very low values of the thermal conductance (about $10^{-7} \mathrm{~W} \cdot \mathrm{K}^{-1}$ ) could be measured in the $2 \mu \mathrm{m}$ wide suspended bridges. The resulting bolometer responsivity could thus be increased by more than 4 orders of magnitude compared to non-suspended bolometers. Thanks to the low thermal conductance and to the low $1 / \mathrm{f}$ noise (normalized Hooge parameter in the order of $10^{-30} \mathrm{~m}^{3}$ or even below), we could demonstrate that the phonon noise, also known as thermal fluctuation noise, which can be considered as the limit noise source for bolometers, could be dominant. The uncooled LSMO bolometers finally showed record low Noise Equivalent Power of about $10^{-12} \mathrm{~W} \cdot \mathrm{Hz}^{-1 / 2}$ at room temperature.
\end{abstract}

Keywords—low frequency; 1/f noise; bolometer

\section{INTRODUCTION}

A bolometer is a thermal radiation detector, which means that the incoming radiation is measured by the temperature elevation caused by its absorption. In order to fabricate high signal-to-noise ratio detectors, one has to reduce the thermal conductance between the active area and the substrate in order to increase the temperature increase, and also choose a material with a large variation of electrical resistance with temperature [1]. The reduction of thermal conductance can be achieved by removing the substrate below the active area using silicon micromachining for example. Due to the reduced heated volume, the thermal response time is interestingly kept low.

The manganite composition $\mathrm{La}_{0.7} \mathrm{Sr}_{0.3} \mathrm{MnO}_{3}$ (LSMO) is a promising candidate for a new generation of uncooled bolometers as it is showing a large electrical resistance change at the metal-to-insulator (M-I) transition. This variation is measured by the Temperature Coefficient of Resistance TCR $=$ $(1 / \mathrm{R}) /(\mathrm{dR} / \mathrm{dT})$. TCR in LSMO is about $0.02 \mathrm{~K}^{-1}$ at temperatures close to $300 \mathrm{~K}$ whereas is about $0.004 \mathrm{~K}^{-1}$ in platinum for example. Moreover LSMO thin films exhibit very low intrinsic noise at $300 \mathrm{~K}$ on $\mathrm{SrTiO}_{3}$ (STO) substrates, which could enable to fabricate low noise bolometers [2].
We previously showed that the fabrication of epitaxial LSMO/STO suspended microbridges using ion etching followed by reactive ion etching (RIE) of the silicon substrate, without degrading the electrical properties of LSMO is possible [3]. In this paper, we show how to get phonon noise limited uncooled bolometers using epitaxial LSMO/STO suspended microbridges. Some bolometer background is given in section II. In section III, we present the experimental details. The electrical and thermal properties of the suspended LSMO/STO microbridges are described in Section IV. Low noise measurements of the uncooled bolometers and NEP analysis are presented in section IV. Finally section $\mathrm{V}$ gives the conclusion.

\section{BOLOMETRY BACKGROUND}

One can write the expression of the optical responsivity $\mathfrak{R}_{\mathrm{V}}$ (expressed in $\mathrm{V} \cdot \mathrm{W}^{-1}$ ) of a bolometer of electrical resistance $\mathrm{R}$ from the equation of energy conservation of the received (both electrical and optical) and evacuated power. It is defined as the output voltage $\Delta \mathrm{V}(\mathrm{f})$ per incoming radiation power $\Delta \mathrm{P}(\mathrm{f})$, where $\mathrm{f}$ is the modulation frequency of the incoming radiation power, and it can be written as in the case of a constant current biased bolometer:

$$
\mathfrak{R}_{\mathrm{V}}(\mathrm{f})=\frac{\Delta \mathrm{V}(\mathrm{f})}{\Delta \mathrm{P}(\mathrm{f})}=\frac{\eta \cdot \mathrm{I}}{\left(\mathrm{G}-\mathrm{I}^{2} \cdot \frac{\mathrm{dR}}{\mathrm{dT}}\right) \cdot \sqrt{1+(2 \cdot \pi \mathrm{f} \tau)^{2}}} \cdot \frac{\mathrm{dR}}{\mathrm{dT}}
$$

where $\eta$ is the absorption coefficient (dimensionless), $I$ is the bias current (in A), $\mathrm{G}$ is the thermal conductance of the device (in $\mathrm{W} \cdot \mathrm{K}^{-1}$ ), $\tau=\mathrm{C} / \mathrm{G}$ is the thermal time constant (in $\mathrm{s}$ ), $\mathrm{C}$ is the thermal capacitance (in $\mathrm{J} \cdot \mathrm{K}^{-1}$ ).

In the meanwhile, in order to keep thermal time constant low, small thermal capacitance is required. Suspended thin films are then a good solution for achieving both high responsivity and low thermal reponse time (i.e. high cutt-off frequency), by reducing simultaneously both $\mathrm{G}$ and $\mathrm{C}$.

The conventional way of specifying the noise of a bolometer is to use its noise equivalent power NEP which can be interpreted as the input signal power that produces a signalto-noise ratio of unity at the output of a given detector at a 
modulation frequency, and effective noise bandwidth; it is the minimum detectable power per square root bandwidth.

The total equivalent power spectral density of a bolometer is determined by the Johnson noise, the phonon noise, the photon noise, the readout noise and the excess noise.

The photon noise is due to the background radiation received by the bolometer. At $300 \mathrm{~K}$, the photon noise is negligible.

The phonon noise is caused by the phonons passing from the detector over the thermal conductance to the substrate and is calculated from the temperature fluctuations inside the system $[4,5]$. The phonon noise power spectral density is given by:

$$
\mathrm{S}_{\mathrm{V}-\text { phonons }}^{2}=\left(4 \cdot \mathrm{k}_{\mathrm{B}} \cdot \mathrm{T}^{2} \cdot \mathrm{G}\right) \cdot \mathfrak{R}_{\mathrm{V}}^{2}(\mathrm{f}) \propto \frac{\mathrm{G} \cdot \mathrm{I}^{2}}{\left(\mathrm{G}-\mathrm{I}^{2} \cdot \frac{\mathrm{dR}}{\mathrm{dT}}\right)^{2}}
$$

It can be reduced by lowering the thermal coupling of the bolometer to the thermal bath, i.e. the thermal conductance $\mathrm{G}$, which is also desired for an increased responsivity.

The Johnson noise is due to random fluctuations of the electron inside the bolometer. The Johnson noise power spectral density is given by:

$$
\mathrm{S}_{\mathrm{V}-\mathrm{J}}^{2}=4 \cdot \mathrm{k}_{\mathrm{B}} \cdot \mathrm{T} \cdot \mathrm{R}
$$

This noise contribution to the NEP can be reduced by increasing the responsivity of the bolometer, by reducing the electrical resistance value or by reducing the temperature.

The read-out noise is due to the electronics set-up. Using a dedicated home made current source and a dedicated home made voltage amplifier, this contribution could be made negligible compared to others.

The excess noise can be described by the following Hooge empirical relation generally verified in normal metals. Its contribution in noise power spectral density term is given by:

$$
\mathrm{S}_{\mathrm{V}-1 / \mathrm{f}}^{2}=\frac{\alpha_{\mathrm{H}}}{\mathrm{n}} \cdot \frac{\mathrm{V}^{2}}{\Omega \cdot \mathrm{f}}
$$

where $\alpha_{H}, n, \Omega$ and $\mathrm{f}$ are respectively the dimensionless Hooge parameter, the charge carrier density $\left[\mathrm{m}^{-3}\right]$, the sample volume $\left[\mathrm{m}^{3}\right]$ and the measuring frequency $[\mathrm{Hz}]$. The lowfrequency noise brings information about the film processing technique quality and the charge transport properties.

Since the noise sources are uncorrelated, the resulting power spectral density is the quadrature sum of all contributions.

In order to achieve the lowest possible NEP, the bolometer parameters must be optimized for the operating conditions in order to get high responsivity and low total power noise spectral density (i.e. high signal-to-noise ratio).

\section{EXPERIMENTAL DETAILS}

We used $75 \mathrm{~nm}$ thick LSMO films grown on $20 \mathrm{~nm}$ thick STO buffer layers on Si (001) substrates by reactive MBE [6]. A fabrication process based on the silicon substrate removal by RIE lead to the successful fabrication of suspended LSMO strips with undegraded electrical properties. The suspended bridges were fabricated by using the isotropic nature of the reactive ion etching of the silicon in SF6 gas as described in [3].

A large number of suspended LSMO strips of various width ( 2 or $4 \mu \mathrm{m})$, length (50 to $200 \mu \mathrm{m}$ ), as well as suspended strips in parallel in order to form square suspended surfaces have been characterized. In this paper, two kinds of structures are presented: $2 \mu \mathrm{m}$ wide LSMO suspended microbridges as well as non suspended LSMO line patterned on the same $\mathrm{Si}$ substrate.

The electrical resistance of four $2 \mu \mathrm{m}$ wide LSMO suspended microbridges as well as of the non suspended LSMO line was measured as a function of temperature in a four probe configuration. A prober with a variable temperature stage (LAKESHORE TTP4) to heat the sample up to around $360 \mathrm{~K}$ in a primary vacuum atmosphere, and a Semiconductor Parametric Analyzer (HP3562A) to plot the current-voltage I(V) characteristics have been used.

The bolometer structures were current biased using a homemade quasi-ideal DC current source with negligible noise and high output impedance [7]. The voltage and current measurements were made with the four-wire technique. Measurements of the optical responsivity versus frequency were made using a spectrum analyzer (HP3562A), and a homemade voltage amplifier circuit.

\section{RESULTS}

The electrical resistance values were deduced from the slope of the I-V characteristics after having checked the linear nature of the curves (i.e., an ohmic behavior). Fig. 1 shows the electrical resistance versus temperature characteristics of the four suspended bridges as well as of the non suspended line. The electrical resistivity at $295 \mathrm{~K}$ of the non suspended line is about $3.1 \mathrm{~m} \Omega \cdot \mathrm{cm}$ which is close to bulk value of LSMO [1]. The electrical resistivity mean value at $295 \mathrm{~K}$ of the suspended bridges is $2.5 \mathrm{~m} \Omega \cdot \mathrm{cm}$.

Values of the maximum of TCR are around $0.02 \mathrm{~K}^{-1}$. The temperature of the maximum of TCR is around $320 \mathrm{~K}$ for all the suspended microbridges and the non suspended structure. These results demonstrate that our fabrication process did not degrade the LSMO. 


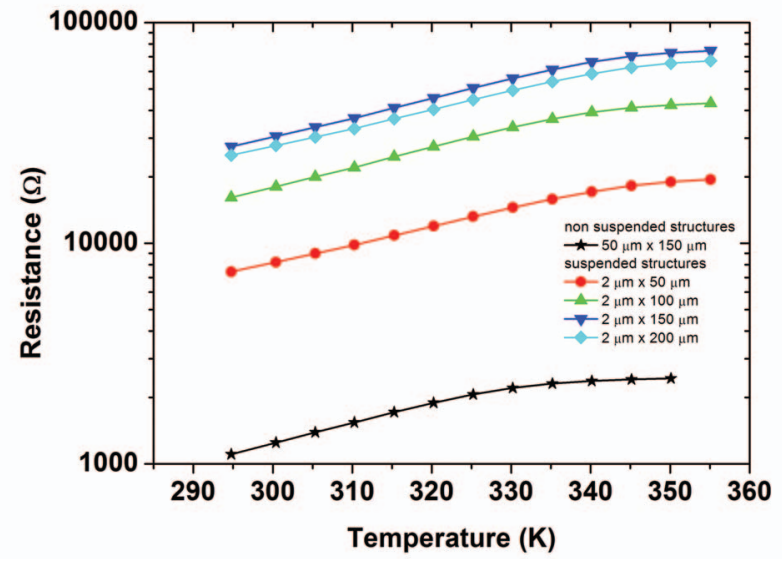

Fig. 1.Electrical resistance as a function of the temperature of four $2 \mu \mathrm{m}$ suspended bridges with different lengths $(50 \mu \mathrm{m}, 100 \mu \mathrm{m}, 150 \mu \mathrm{m}$ and $200 \mu \mathrm{m})$ and of a non suspended structure $(50 \mu \mathrm{m}$ wide, $150 \mu \mathrm{m}$ long $)$

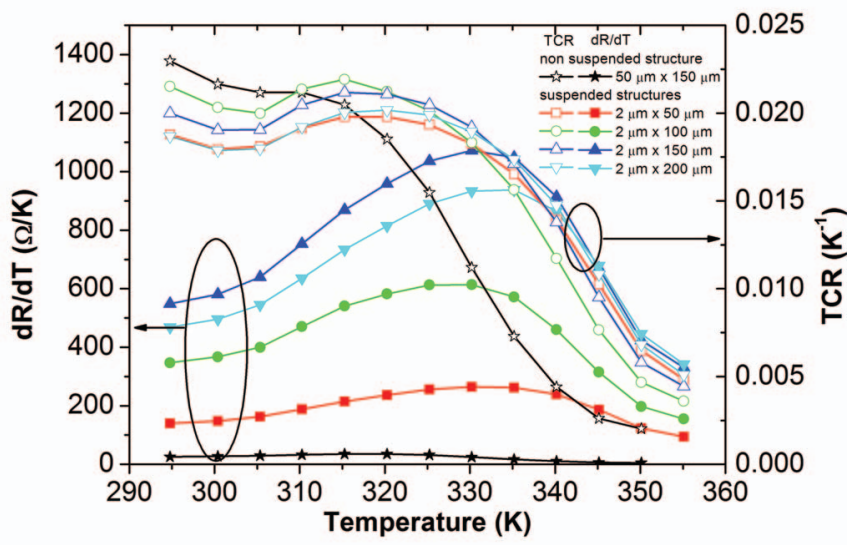

Fig. 2.dR/dT (left axis) and TCR (right axis) as a function of the temperature of four $2 \mu \mathrm{m}$ suspended bridges with different lengths $(50 \mu \mathrm{m}, 100 \mu \mathrm{m}$, $150 \mu \mathrm{m}$ and $200 \mu \mathrm{m})$ and of a non suspended structure (50 $\mu \mathrm{m}$ wide, $150 \mu \mathrm{m}$ long)

Thermal conductance can be calculated as the thermal conductance through the mechanical support only, which is the LSMO/STO self-supported microbridge itself. In the case of uniform heat production $\mathrm{P}$ received by the bridge of electrical resistance $\mathrm{R}$, we can define the average temperature rise $\Delta \mathrm{T}$ and we can show from the equation of heat flow in solids that the thermal conductance is $[8,9]$ :

$$
\mathrm{G}=\frac{12 \cdot \mathrm{w}}{1} \cdot\left(\mathrm{k}_{\mathrm{LSMO}} \cdot \mathrm{t}_{\mathrm{LSMO}}+\mathrm{k}_{\mathrm{STO}} \cdot \mathrm{t}_{\mathrm{STO}}\right)
$$

where 1 and $\mathrm{w}$ are the length and the width of the suspended bridge, $\mathrm{k}_{\mathrm{LSMO}}, \mathrm{k}_{\mathrm{STO}}$ and $\mathrm{t}_{\mathrm{LSMO}}, \mathrm{k}_{\mathrm{STO}}$ are the thermal conductivity and thickness of each layer constituting the suspended bridge.

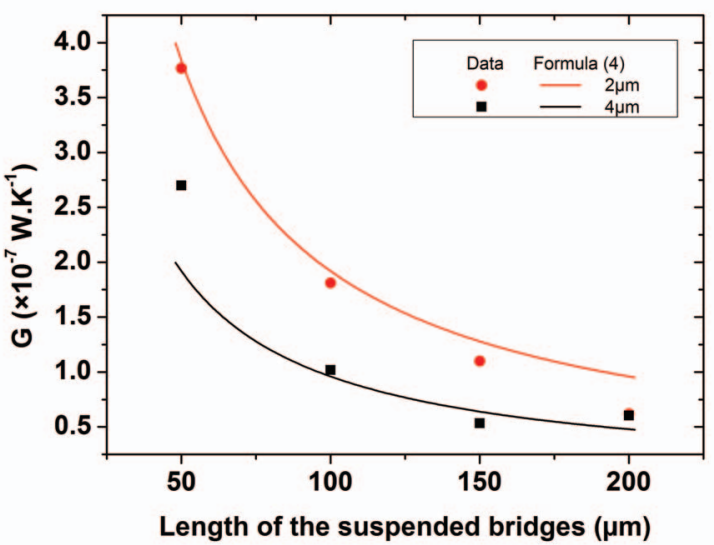

Fig. 3. Thermal conductance of suspended bridges at $300 \mathrm{~K}$

The thermal conductance of suspended strips was measured at $300 \mathrm{~K}$ (Fig. 3). One can see that the measured $\mathrm{G}$ values are very close to the calculated ones using (4) and thermal conductivity values of LSMO and STO $\left(2.5\right.$ and $11.6 \mathrm{~W} \cdot \mathrm{m}^{-1} \cdot \mathrm{K}^{-}$ ${ }^{1}$, respectively [10-13]).

The measured values are more than 4 orders of magnitude lower than the thermal conductance of non-suspended strips [14]. The resulting responsivity is thus increased by more than 4 orders of magnitude compared to non-suspended bolometers (see (1)).

In our experiment, the measured noise power spectral densities consist of $1 / \mathrm{f}$ noise at low frequency and white noise (up to $10 \mathrm{kHz}$ ). Figure 4 show the noise power spectral densities of the non suspended bridge as well as of the suspended bridges at $300 \mathrm{~K}$. Noise spectra typically consist of two parts: a low frequency noise and the white part. No lorentzian component was observed.

In case of the non suspended bridge, the white noise is independent of current value and frequency, which can be estimated by Johnson noise. After checking the quadratic dependence of $\mathrm{S}_{\mathrm{V}}$ with the sample voltage the normalized Hooge parameter $\alpha_{\mathrm{H}} / \mathrm{n}$ is estimated. For $1 / \mathrm{f}$ noise, its value is very low $\left(6.6 \times 10^{-30} \mathrm{~m}^{3}\right)$ and is comparable to published values [2].

In case of the suspended bridges, $1 / \mathrm{f}$ noise is also very low $\left(7 \times 10^{-31} \mathrm{~m}^{3}\right)$. The white noise is dependent of current bias. It is not explained by the Johnson noise which has to be independent of the current value. The dependence is linked to the phonon noise indeed. The noise power spectral density due to phonon noise is quadratic dependent of the current bias and is inversely proportional to $\mathrm{G}$. As the thermal conductance $\mathrm{G}$ is very low, phonon noise becomes dominant in the middle frequency range. This behavior could be observed since the excess noise is very low for LSMO compared to other thermoresistive materials used at $300 \mathrm{~K}$ [2]. 

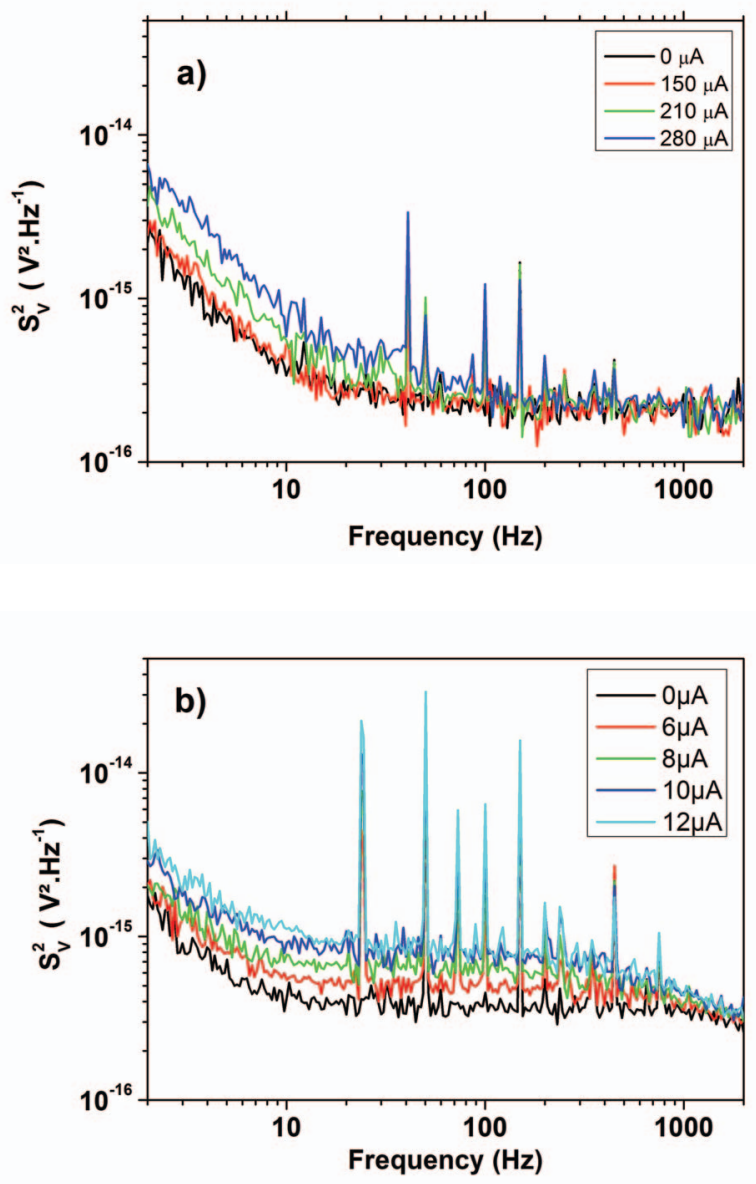

Fig. 4. Noise power spectral densities measured at $300 \mathrm{~K}$ for different bias currents: a) a $50 \mu \mathrm{m}$ wide $150 \mu \mathrm{m}$ long non suspended bridge and b) a $2 \mu \mathrm{m}$ wide $50 \mu \mathrm{m}$ long suspended bridge

The uncooled LSMO bolometers finally showed record low Noise Equivalent Power of about $10^{-12} \mathrm{~W}_{\mathrm{H}} \mathrm{Hz}^{-1 / 2}$ at room temperature which is the state of the art for uncooled thermal detector.

\section{REFERENCES}

[1] A. Urushibara, Y. Moritomo, T. Arima, A. Asamitsu, G. Kido, Y. Tokura, Phys. Rev. B, 51, 14103, 1995

[2] L. Méchin, S. Wu, B. Guillet, P. Perna, C. Fur, S. Lebargy, C. Adamo, D.G. Schlom, J.M. Routoure, J. Phys. D: Appl. Phys. - Fast Track Communication, 46, 2020012013

[3] S. Liu, B. Guillet, A. Aryan, C. Adamo, C. Fur, J.-M. Routoure, F. Lemarié, D.G. Schlom, L. Méchin, Microelectron. Eng., 111, 101, 2013

[4] J.C. Mather, Appl. Opt. (21): 1125-1129, 1982

[5] J.C. Mather, Appl. Opt. (23) 584, 1984

[6] L. Méchin, C. Adamo, S. Wu, B. Guillet, S. Lebargy, C. Fur, J.-M. Routoure, S. Mercone, M. Belmeguenai, and D. G. Schlom, Phys. Status Solidi A, 209, 1090, 2012

[7] J.-M. Routoure, D. Fadil, S. Flament, and L. Méchin, Proc. 19th International Conference on Noise and Fluctuations; ICNF 2007, AIP Conference Proceedings, vol. 922, no. 1, pp. 419-424, 2007

[8] D. P. Neikirk, Wayne W. Lam, and D. B. Rutledge, Int. J. Infrared Milli., Vol. 5, No. 3, 1984

[9] L. Méchin, J.C. Villégier, D. Bloyet, J. Appl. Phys., 81, (10), 7039, 1997

[10] L.M. Wang, J.-H. Lai, J.-I. Wu, J. Appl. Phys., 102, 023915, 2007

[11] I. El-Kassab, A.M. Ahmed, P. Mandal, K. Barner, A. Kattwinkel, U. Sondermann, Physica B, 305, 233, 2001

[12] H. Muta, K. Kurosaki, S. Yamanaka, J. Alloys Compd., 392, 306, 2005

[13] D.-W. Oh, J. Ravichandran, C.W. Liang, W. Siemons, B. Jalan, C.M. Brooks, M. Huijben, D.G. Schlom, S. Stemmer, L.W. Martin, A. Majumdar, R. Ramesh, D.G. Cahill, Appl. Phys. Lett., 98, 221904, 2011

[14] A. Aryan, B. Guillet, J.M. Routoure, C. Fur, P. Langlois, L. Méchin, Appl. Surf. Science, 326, 204, 2015 